\title{
The multiple dependence of the velocity distributions of granular gases on the simulation conditions
}

\author{
Antony M.M. Polito ${ }^{\mathrm{a}, *}$, Annibal Figueiredo ${ }^{\mathrm{a}}$, Luis S. Costa ${ }^{\mathrm{a}}$, Tarcísio M. da Rocha \\ Filho $^{\mathrm{a}}$, Frederico V. Prudente ${ }^{\mathrm{b}}$ \\ anstituto de Física, Universidade de Brasilia, 70919-970 - Brasilia, Brazil \\ ${ }^{\mathrm{b}}$ Instituto de Física, Universidade Federal da Bahia, 40210-340 Salvador, Brazil
}

Available online 19 May 2006

\begin{abstract}
In this work we study the dissipative dynamics of a system of smooth hard spheres with a constant restitution coefficient and small number of particles in the homogeneous cooling regime. We focus on the velocity distribution from simulations in the molecular dynamics approach. The main goal of this paper is to study the multiple dependence of these stationary states on the number of particles, density regimes and boundary conditions for a fixed restitution coefficient and the existence of a limit state in the thermodynamic limit. For this we present a methodology based on the application of the characteristic function technique and the $W$ function, introduced by Lévy to measure the distance of distributions from the Gaussian. (C) 2006 Elsevier B.V. All rights reserved.
\end{abstract}

Keywords: Granular systems; Homogeneous cooling; Characteristic function

\section{The physical system description and their simulation}

We consider a three-dimensional system composed by $N$ hard spheres with diameter $a$ and unit mass in a cubic box of unit volume. The particles interact through instantaneous collisions with a constant restitution coefficient $\alpha$ [1,2]. Only the change in the relative velocity along the radial direction of a collision is considered here for the energy decrease of the system. We are interested essentially in small systems in the homogeneous cooling state (HCS) [1,2], in such a way the number $N$ of particles is at most of the order $10^{3}$.

The simulations are performed using an event-driven molecular dynamics algorithm [3], with two distinct boundary conditions: periodic and reflexive. Throughout the paper all length measures are given in units of the box size, including the particle diameter and the mean free path which become dimensionless. The initial conditions is imposed to be spatially homogeneous and the initial velocity distributions is chosen in a class of non-Gaussian distributions given by a truncated Lévy distribution:

$$
f(x)=\frac{1}{2 \arctan (L)} \frac{1}{1+x^{2}} \text { if }-L \leqslant x \leqslant L \text { and } f(x)=0 \text { otherwise, }
$$

where the parameter of truncation $L$ defines the distribution inside the class.

*Corresponding author.

E-mail address: antony@fis.unb.br (A.M.M. Polito). 
Each system will be characterized by a specific boundary and by three parameters: the number of particles $N$, the restitution coefficient $\alpha$, and $\delta=N a^{2}$, a dimensionless factor related to the normalized mean free path given by $l_{0}=1 / \sqrt{2} \pi N a^{2}$. Hence, a fixed value for $\delta=N a^{2}$ is equivalent to an inferior boundary for the normalized mean free path.

\section{The characteristic function and the Lévy's $W$ function}

The characteristic function (CF) $\psi(z)$ associated to the reduced variable $\bar{x}$ is defined as the mean $\langle\exp (\mathrm{i} \bar{x} z)\rangle=(1 / N) \sum_{j=1}^{N} \exp \left(\mathrm{i} z \bar{x}_{j}\right)$ where $N$ is the number of elements of the sample. The CF is interesting because it possess, in each point, global information about the distribution function. In this way, it allows the calculus of the distribution with high degree of accuracy, even in the points that the particle density is very low, as in the tails.

The French mathematician Paul Lévy [4] demonstrated that any CF associated to a reduced variable can be written as $\psi(z)=\exp \left(-z^{2} / 2(1+W(z))\right)$ where the complex valued function $W(z)$, in the following called Lévy's $W$ function, is a continuous function in an open interval $-\varepsilon<z<\varepsilon$ and such that $W(0)=0$. The Gaussian distribution is characterized by $W(z)=0$ for all $z$. Here, we will consider as reduced random variable $c_{x}=\sqrt{2} v_{x} / v_{0}(\tau)$, where $v_{x}$ is the $x$-component of the velocity vector $\mathbf{v}$ and $v_{0}$ is the thermal velocity.

\section{Simulation results}

The first question to be dealt with refers to the convergence of the initial distributions to well-defined stationary states. We implement simulations with the following fixed parameters: number of particles $N=256$, restitution coefficient $\alpha=0.1$ and $\delta=0.7$ and 1.0 , both for reflexive and periodic boundaries. In Fig. 1a we show the $W$ function corresponding to four initial conditions $(L=0.1,6.0,12.0$ and 40.0$)$. Fig. 1b shows the $W$ function for the asymptotic stationary state corresponding to periodic and reflexive boundaries. The figures show the existence of different non-Gaussian asymptotic stationary distributions.

It is important to observe the difference amongst the asymptotic distributions for different boundary choices. The simulations demonstrate that the imposed boundary conditions actually guide the system to different asymptotic distributions, even though all the other parameters of the system are identical.

Another observation refers to the influence of the parameter $\delta$. For each value of $\delta$ a different asymptotic state is obtained, even though the other parameters, $N$ and $\alpha$, and the boundary conditions remain identical. We observe this in Fig. 2 where in each case there is a different asymptotic distribution for $\delta=0.7$ and $\delta=1.0$, both for reflexive and periodic conditions. Simulations carried out with different restitution coefficients show that these features are valid for all range of inelasticities $(0<\alpha<1)$.

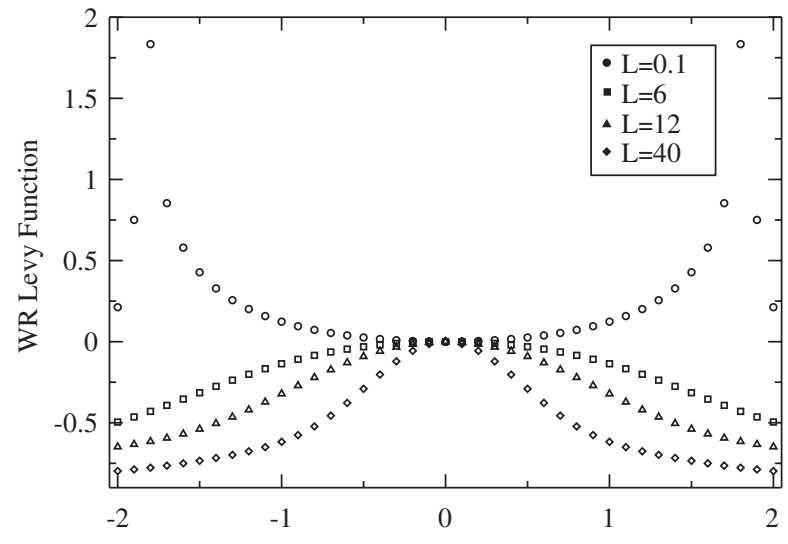

(a)

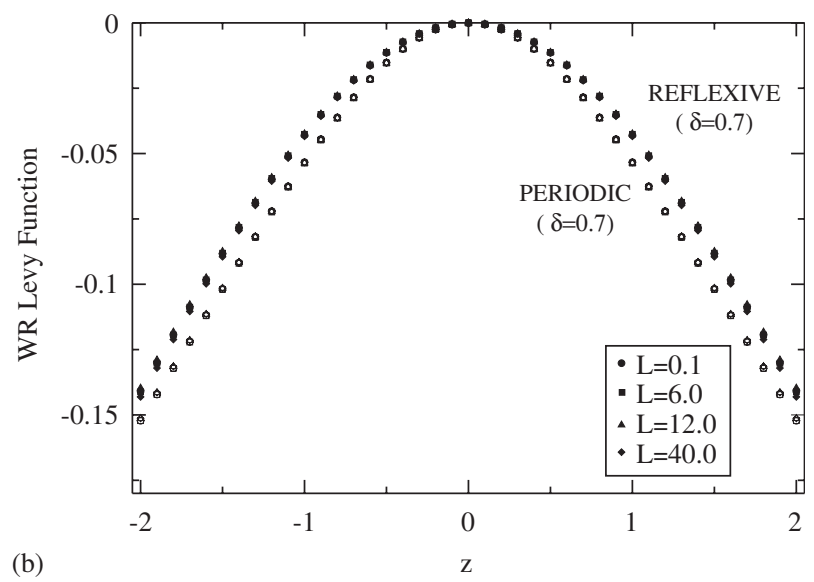

(1)

Fig. 1. (a) The function $W(z, 0)$ corresponding to four initial conditions ( $L=0.1,6.0,12.0$ and 40.0$)$. (b) The asymptotic $W(z)$ function evolved with periodic and reflexive boundaries. For the simulations $N=256, \delta=0.7$ and $\alpha=0.1$. 
The simulations demonstrate that the asymptotic velocity distributions are strongly dependent of the number $N$ of particles, since $\alpha, \delta$ and the boundary conditions are fixed. Then, we investigate the behavior of several systems in the so-called thermodynamic limit $N \rightarrow \infty$, while the parameter $\delta$ remains constant. Fig. 3 shows the existence of different asymptotic states for each choice of $N$. We observe that the increasing of the number of particles $N$ suggests a convergence process of the distributions towards a unique asymptotic nonGaussian state in the thermodynamic limit. Moreover, it is important to stress that our systems never develops clusters or vortices, even though it is known that HCS is unstable under spatial and velocity fluctuations. For this is important to maintain $\delta$ constant and below a certain critical value in the limit process, since the mean free path is the parameter that defines the stability thresholds [5].

On the other side, Fig. 4 shows that the influence of $\delta$ is irrelevant in the thermodynamic limit, since the distributions converge with increasing $N$ to the same state. Besides, the convergence towards the limit state is relatively fast. Nevertheless, the study of cases using periodic boundaries does not lead to same straight conclusions. In this case the influence of the parameter $\delta$ in the splitting of states is still present even with $N=55,296$. This number is already very large when compared to the reflexive case. These differences between the two different boundaries points to a problem in the use of periodic boundaries in simulations. They are very sensitive to the choice of the mean free path of the system, even if the number of particles is large.

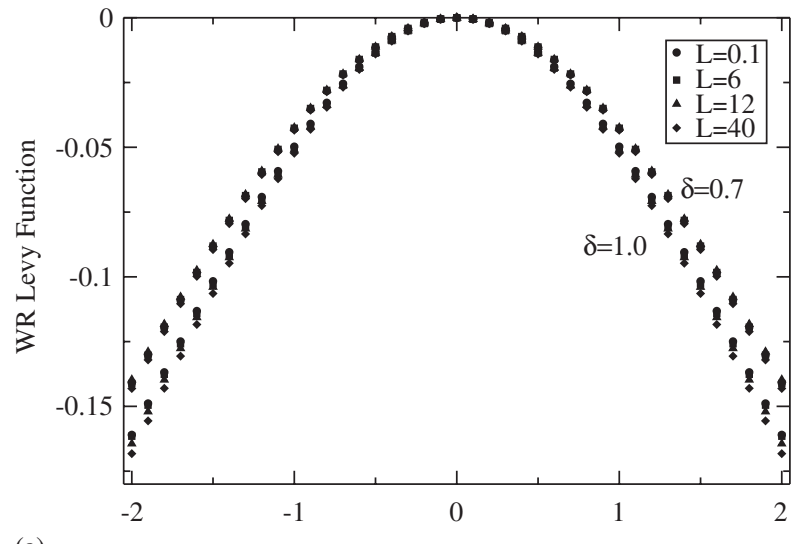

(a)

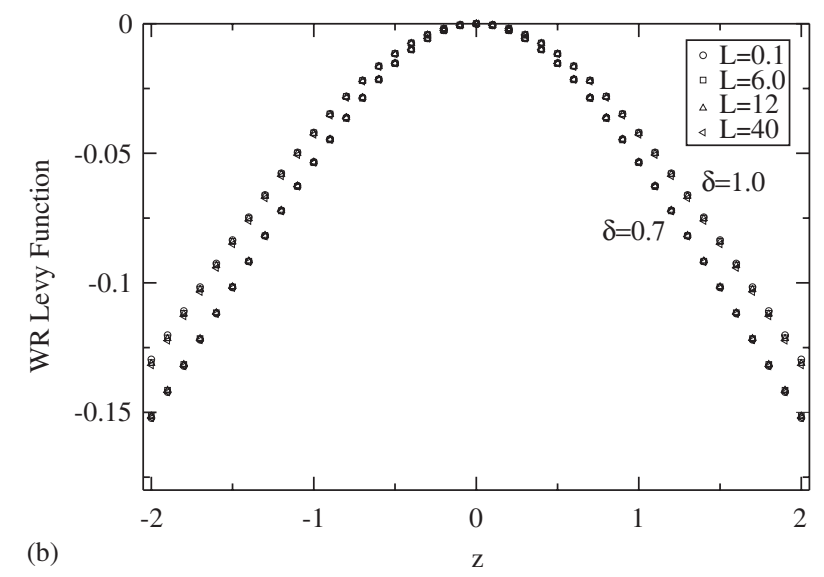

Fig. 2. The asymptotic $W(z)$ function evolved from the above initial conditions: (a) for $\delta=0.7$ and $\delta=1.0$, with reflexive boundary conditions; (b) for $\delta=0.7$ and $\delta=1.0$, with periodic boundary conditions. For the simulations $N=256$ and $\alpha=0.1$.
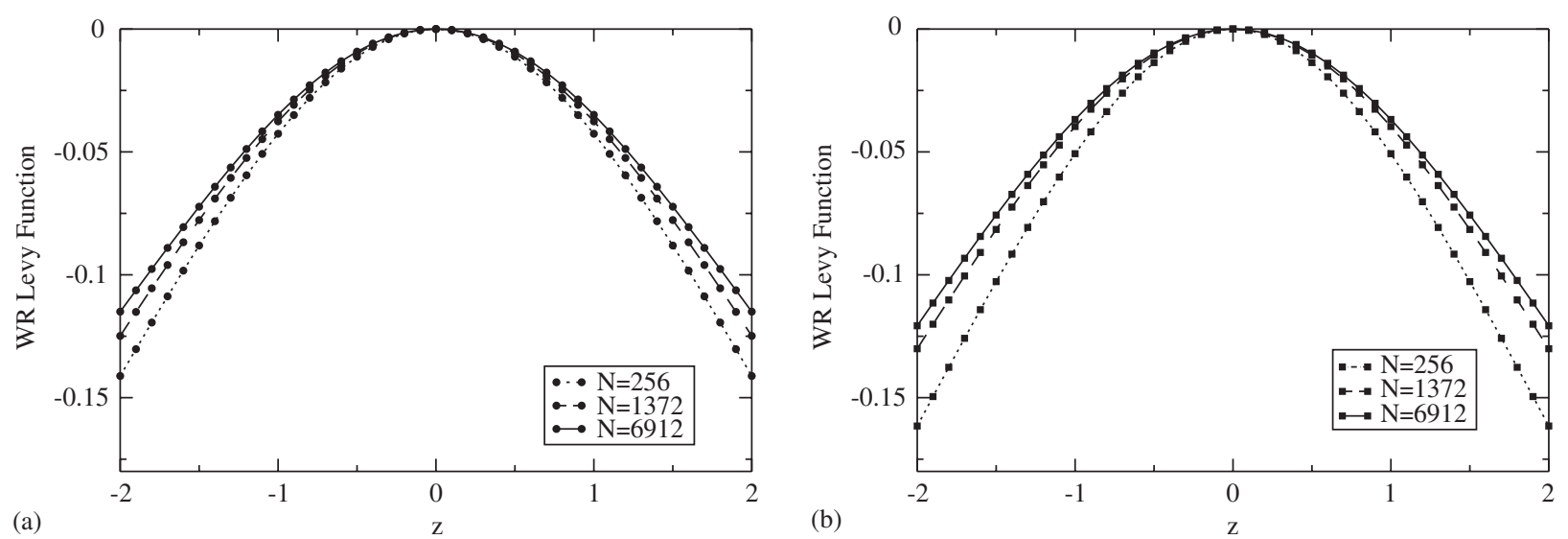

Fig. 3. The asymptotic $W(z)$ function for systems with three different number of particles $(N=256,1372$ and 6912$)$ evolved with reflexive boundaries: (a) for $\delta=0.7$; (b) for $\delta=1.0$. For the simulations $\alpha=0.1$. 

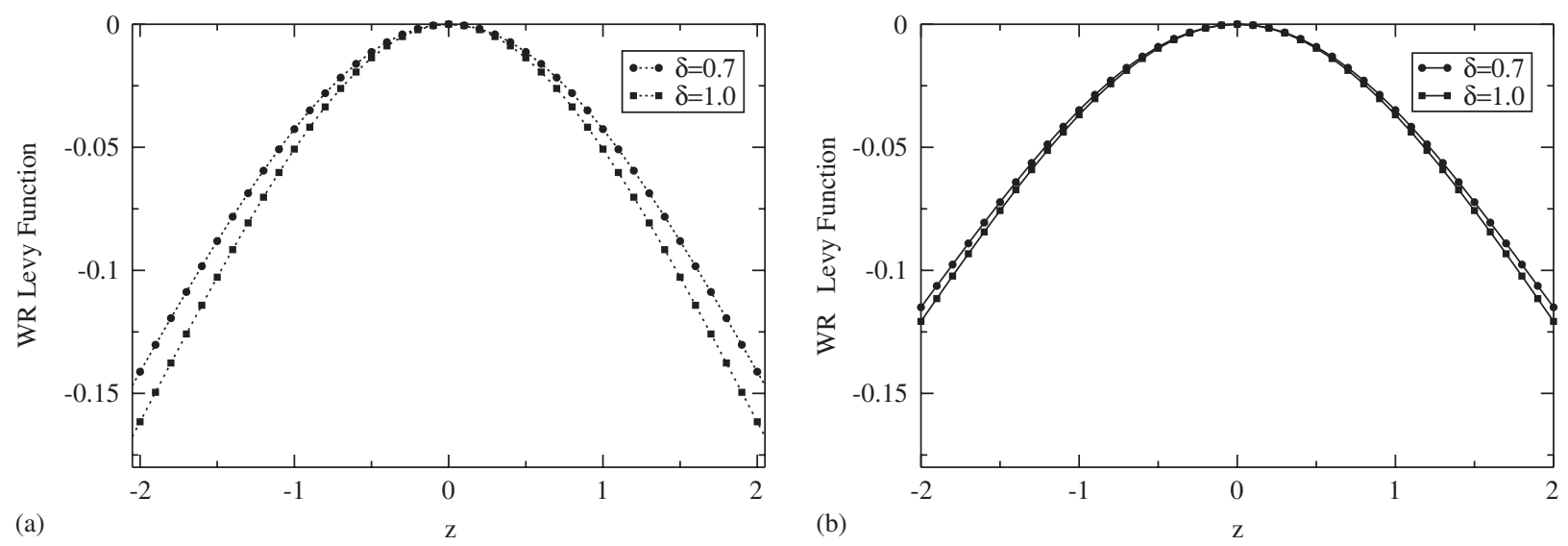

Fig. 4. The asymptotic $W(z)$ function for systems at two different density regimes $(\delta=0.7$ and 1.0) evolved with reflexive boundaries: (a) for $N=256$; (b) for $N=6912$. For the simulations $\alpha=0.1$.

In summary, the use of the $W$ function to deal with simulation data shows that different stationary asymptotic distributions exist and are determined by four independent conditions: the number of particles, the parameter $\delta$ (related to the mean free path), the restitution coefficient and the implemented boundary condition. The Lévy $W$ function allows the splitting and the clear verification of the existence of these different states. At the thermodynamic limit, we conjecture that the stationary distributions converge to a unique limit state, which depends exclusively on the inelasticity parameter.

\section{Acknowledgment}

The authors acknowledge the financial support of FINATEC and CNPq (Brazil).

\section{References}

[1] A. Goldshtein, M. Shapiro, J. Fluid Mech. 282 (1995) 75.

[2] T.P.C. van Noije, M.H. Ernst, Granular Matter 1 (1998) 57.

[3] M.P. Allen, D.J. Tildesley, Computer Simulations of Liquids, Oxford University Press, Oxford, 1997.

[4] P. Lévy, C. R. Acad. Sci. 174 (1922) 1682 (Oeuvres de Paul Lévy, Eléments Aléatoires, vol. 3, Gauthiers-Villars, Paris, 1976).

[5] I. Goldhirsh, G. Zanetti, Phys. Rev. Lett. 70 (1993) 1619. 